\title{
A reação das vítimas ao cyber-hate no Facebook: Contribuições da Teoria da Ação Planejada
}

\author{
Sheyla Fernandes'; Érika Melo²; Marcikele Nascimento; \\ Stelio Junior ${ }^{2}$, Leogildo Alves Freires ${ }^{1}$ e Jesana Silva²; \\ ${ }^{1}$ Docente do Instituto de Psicologia da Universidade Federal de Alagoas, Brasil I \\ sheyla.fernandes@ip.com.br I https://orcid.org/0000-0003-4759-1314 \\ 2 Discentes do Programa de Graduação do Instituto de Psicologia da Universidade
}

Federal de Alagoas, Brasil I erikadsm26@gmail.com; nascimentomarcikele@gmail.com; stelionovaesc@gmail.com; leogildo.freires@ip.ufal.br; jesanapriscila123@gmail.com I https://orcid.org/0000-0001-6045-0327; https://orcid.org/0000-0002-4844-743X https://orcid.org/0000-0001-5271-8098; https://orcid.org/0000-0001-5149-2648; https://orcid.org/0000-0003-0567-3012

Resumo: O cyber-hate é um fenômeno social multifacetado caracterizado pela expressão de insultos e humilhações dirigidos a indivíduos e grupos no cyber-espaço. Trata-se de um fenômeno extremamente danoso que em função do anonimato ou da distância emocional das vítimas, acontece de forma recorrente nos ambientes virtuais. Pouca atenção tem sido direcionada à perspectiva das vítimas sobre as ofensas recebidas. Deste modo, este estudo tem a finalidade de investigar de que forma as vítimas respondem ao cyber-hate no contexto do Facebook. Foi elaborado um questionário a partir dos construtos da Teoria da Ação Planejada (TAP) com o objetivo de analisar as crenças, atitudes e normas acerca da reação das vítimas ao cyber-hate. O questionário foi disponibilizado em páginas do Facebook previamente selecionadas de militância sexual, ativismo étnico-racial, ativismo nordestino e militância política. Contou-se com a colaboração de 30 respondentes. Os dados coletados foram transformados em um corpus textual e analisados pelo software Iramuteq, por meio da Classificação Hierárquica Descendente (CHD). O corpus apresentou o quantitativo de 5540 ocorrências, com 322 formas ativas e um aproveitamento 138 segmentos de texto (ST) dos $172(80,23 \%)$. A CHD apresentou a divisão do conteúdo em 3 classes: Classe 1 ("Crenças Comportamentais"), representando $43,5 \%$ da UCE's e refletindo as vantagens e desvantagens em responder ao cyber-hate; Classe 2 ("Crenças de Controle"), representando $27,5 \%$ UCE's e indicando as facilidades e dificuldades em reagir ao cyberhate; e Classe 3 ("Crenças Normativas"), representando $28,99 \%$ UCE's e destacando a influência de terceiros nas respostas ao cyber-hate. Os resultados permitiram concluir que as crenças exercem um papel central nas formas de reação ao cyber-hate nos ambientes virtuais. A base teórica e conceitual da TAP se constituiu como um pertinente dispositivo para a investigação deste fenômeno no âmbito do Facebook, corroborando com estudos recentes acerca da intolerância digital no contexto brasileiro.

Palavras-chave: Cyber-hate; Intolerância; Facebook; Redes Sociais Online.

\section{Victims' Reaction to Cyber-hate on Facebook: Contributions from the Theory of} Planned Action

Abstract: Cyber-hate is a multifaceted social phenomenon characterized by the expression of insults and humiliations directed at individuals and groups in cyber-space. It is an extremely damaging phenomenon that, due to the anonymity or emotional distance of the victims, happens on a recurring basis in virtual environments. Little attention has been directed to the victims' perspective on the offenses received. Thus, this study aims to investigate how victims respond to cyber-hate in the context of Facebook. A questionnaire was elaborated based on the Theory of Planned Action (TAP) constructs with the objective of analyzing the beliefs, attitudes and norms about the response with cyber-hate. The questionnaire was made available on Facebook pages previously selected for sexual activism, ethnic-racial activism, northeastern activism and political activism. It counted on the collaboration of 30 respondents. The collected data were transformed into a textual corpus and analyzed by the Iramuteq software, using the Descending Hierarchical Classification (CHD). Results: The corpus presented a total of 5540 occurrences, with 322 active forms and 138 text segments (ST) out of $172(80.23 \%)$. CHD presented the division of content into 3 classes: Class 1 ("Behavioral Beliefs"), representing $43.5 \%$ of UCE's and reflecting the advantages and disadvantages of responding to cyber-hate; Class 2 ("Control Beliefs"), representing 27.5\% UCE's and indicating the facilities and difficulties in facing cyber-hate; and Class 3 ("Normative Beliefs"), representing 28.99\% UCE's and highlighting the influence of third parties in the fight against cyber-hate. The results allowed to conclude that beliefs play a central role in the forms of reaction to cyber-hate in virtual environments. The theoretical and conceptual basis of TAP constitutes itself as a pertinent device for the investigation of this phenomenon in the scope of Facebook, corroborating with recent studies about digital intolerance in the Brazilian context.

Keywords: Cyber-hate; Intolerance; Facebook; Online Social Networks. 


\section{Introdução}

O Facebook, uma das Redes Sociais Online (RSO) mais acessadas do mundo, em janeiro de 2021, somente no Brasil, apresentou uma média nacional de acessos de mais de 150 milhões. Estima-se que em 2021, um terço da população mundial esteja conectada ao Facebook (Statista, 2021).

No ambiente das RSO, as interações sociais são mediadas por aparelhos como computador, celular e tablet, possibilitando que os indivíduos fiquem protegidos por uma tela, e por meio dela, experienciem livremente uma diversidade de formas de interação. A interação virtual, apesar de apresentar um conjunto de aspectos positivos, pautada na liberdade de expressão, vem resultando em agravos invasivos ao espaço do outro, e até na possibilidade de expressão de discursos de ódio (Zenha, 2018). Tais condutas contam com a garantia de anonimato e conforto em atacar outros indivíduos em função de diversos critérios, como a cor, origem geográfica, etnia, orientação sexual, gênero, religião, idade e/ou deficiência, por exemplo (Rocha \& Mendes, 2020). Este tipo de comportamento tem sido nomeado de cyber-hate, e se caracteriza como um fenômeno social multifacetado, que, segundo Silva \& Botelho-Francisco (2020), ocorre em diversas redes sociais online atingindo direta ou indiretamente pessoas e/ou grupos, sendo expresso por meio de insultos, discriminação e humilhações a indivíduos no cyber-espaço.

Dada a relevância do Facebook para essa problemática, o presente estudo foca sua atenção na investigação da reação das vítimas frente ao discurso de ódio propagado no Facebook (Silva \& Botelho-Francisco, 2020). Para tanto, tomou-se a Teoria da Ação Planejada (TAP) como aporte teórico-metodológico para compreender de que forma as vítimas de cyber-hate reagem às ofensas digitais.

Segundo Ajzen e Fishbein (2005), a TAP tem como objetivo compreender como ocorre a intenção comportamental da qual as ações são derivadas. Nesse sentido, para predizer e explicar as escolhas comportamentais, três tipos de crenças são empregadas: as crenças comportamentais, formadas pelas atitudes em relação ao comportamento específico e relacionadas à possibilidade de sua emissão; as crenças normativas referentes à pressão social para realizar a ação e as crenças de controle, referentes ao grau de controle que a pessoa percebe que possui para a realização do comportamento (Fishbein \& Ajzen, 2011; Júnior, 2018). Essas crenças constituem os construtos que formam o modelo da TAP, (1) crenças comportamentais, relacionadas à atitude; (2) crenças normativas, associadas à norma subjetiva; (3) crenças de controle, associadas ao controle comportamental percebido (Ajzen \& Driver, 1991; Ajzen, 1991). Portanto, de acordo com Fishbein e Ajzen (2010), a partir do conhecimento das crenças, o pesquisador consegue criar associações causais a fim de compreender e explicar comportamentos específicos, que no presente estudo, consistirá na reação dos internautas ao cyber-hate.

\section{Metodologia}

\subsection{Seleção do Material e Procedimentos Adotados}

Inicialmente, junto ao site Facebook (http://Facebook.com.br/), foram selecionadas páginas relacionadas à militância sexual, ativismo étnico-racial, ativismo nordestino e militância política. Os critérios elaborados para a seleção das páginas foram: 1) discutir questões referentes aos eixos de investigação deste estudo e 2) apresentar o quantitativo mínimo de dez mil curtidas/seguidores. 
Após a seleção das páginas e objetivando realizar uma análise qualitativa das crenças das vítimas, elaborou-se um questionário semiestruturado composto por 17 questões formuladas a partir dos constructos da TAP: crenças comportamentais (vantagens e desvantagens em reagir ao cyber-hate), crenças normativas (aprovação e desaprovação das pessoas e grupos em relação a reagir ao cyber-hate) e crenças de controle (facilidades e dificuldades percebidas para reagir ao cyber-hate), disponível para ser respondido via interface Google Forms. Além do questionário, foi anexado o Termo de Consentimento Livre e Esclarecido, ambos divulgados nas páginas previamente selecionadas no período temporal de 22 de julho a 22 de agosto de 2020. Com a finalização do período de coleta, apenas os participantes cujos questionários e Termos de Consentimento estavam completamente preenchidos foram considerados para a fase de análise de dados. Segundo, Francis et al. (2004), o número mínimo de participantes para realizar uma pesquisa com base na TAP é de 25 pessoas. Contou-se com a participação de 30 respondentes, 14 do sexo feminino (46,7\%), e 16 do sexo masculino (53,3\%); com faixa etária entre 18 e 31 anos.

\subsection{Organização do Corpus Textual e Análise dos Dados}

$\mathrm{Na}$ sequência, as respostas foram coletadas, transformadas em um único arquivo textual e inseridos no software gratuito OpenOffice Writer, esse software está inserido no pacote OpenOffice e possibilita a incorporação de arquivos e a leitura de relatórios e resultados (Camargo \& Justo, 2016). Nesta plataforma os dados foram revisados e salvos considerando a formatação Unicode (UTF-8). Na construção do corpus foi preservado o anonimato de todos os respondentes.

O corpus textual foi analisado através do software de acesso livre Iramuteq (Interface de $R$ pour les Analyses Multidimensionnelles de Textes et de Questionnaires), produzido em linguagem Python (www.python.org) e ancorado no software estatístico R ( $R$ Development Core Team). Iramuteq é um instrumento de processamento de dados e apresenta uma variedade de análise lexical (Souza, Wall, Thuler, Lowen \& Peres, 2018). Dentre as diferentes análises textuais disponíveis no Iramuteq, foi utilizada a Classificação Hierárquica Descendente (CHD).

A análise lexical por meio da CHD foi escolhida como estratégia de análise de dados pois tem por objetivo formar classes a partir dos segmentos dos textos (ST), para agrupá-los através do vocábulo e da frequência que as palavras aparecem no texto (Camargo \& Justo, 2016). A CHD é uma análise multivariada que gera um dendograma produzido a partir de um subagrupamento realizado pela ferramenta para construção de classes estáveis. A partir desse resultado é possível verificar aproximações e afastamentos entre as classes e realizar interpretações relacionadas à composição das mesmas (Ramos, Lima \& AmaralRosa, 2018).

\section{Resultados}

Os dados foram organizados de modo a revelarem alguns aspectos da interação dos usuários com as páginas de militância selecionadas. Dos 30 respondentes, 46,7\% curtem, seguem ou fazem parte de alguma página, grupo ou movimento online de militância sexual, ativismo étnico-racial, ativismo nordestino ou militância política, enquanto $53,3 \%$, apesar de responderem ao questionário divulgado nas páginas, salientaram que não participavam ativamente desse tipo de sítio no cyber-espaço. É importante destacar que a maioria dos participantes $(83,3 \%)$ informou ter sofrido ofensas nos ambientes digitais, bem como, expressou algum tipo de reação. Destes, $66 \%$ referiram ter ignorado os insultos, $23 \%$ denunciaram e $11 \%$ responderam de forma ofensiva. Dentre as justificativas para expressar algum tipo de reação, diversas explicações foram evocadas simultaneamente: por discordância, por causar estresse, pela impunidade dos agressores, para manter a dignidade ou por estarem atentos aos incentivos expressos na mídia sobre a necessidade de denúncia de crimes cibernéticos. 
Já aqueles que não apresentaram reações, justificaram indicando medo, insegurança ou a tentativa de manutenção do equilíbrio emocional, uma vez que reagir não seria percebido como uma alternativa eficaz para alterar o cenário.

O procedimento seguinte, a análise da CHD, considerando as respostas integralmente, apresentou a separação do corpus em 30 unidades de textos iniciais, divididos em 172 segmentos de textos, elencados em 839 formas distintas, sendo 5540 ocorrências e 322 formas ativas. O corpus obteve um aproveitamento 138 ST dos $172(80,23 \%)$, a CHD apresentou a divisão do conteúdo em 3 classes, nas quais formaram 2 eixos para a discussão dos resultados (Fig. 1). Pode-se observar que a primeira partição gerou a classe 3 em oposição às classes 1 e 2. Posteriormente, houve a divisão entre essas duas últimas classes.

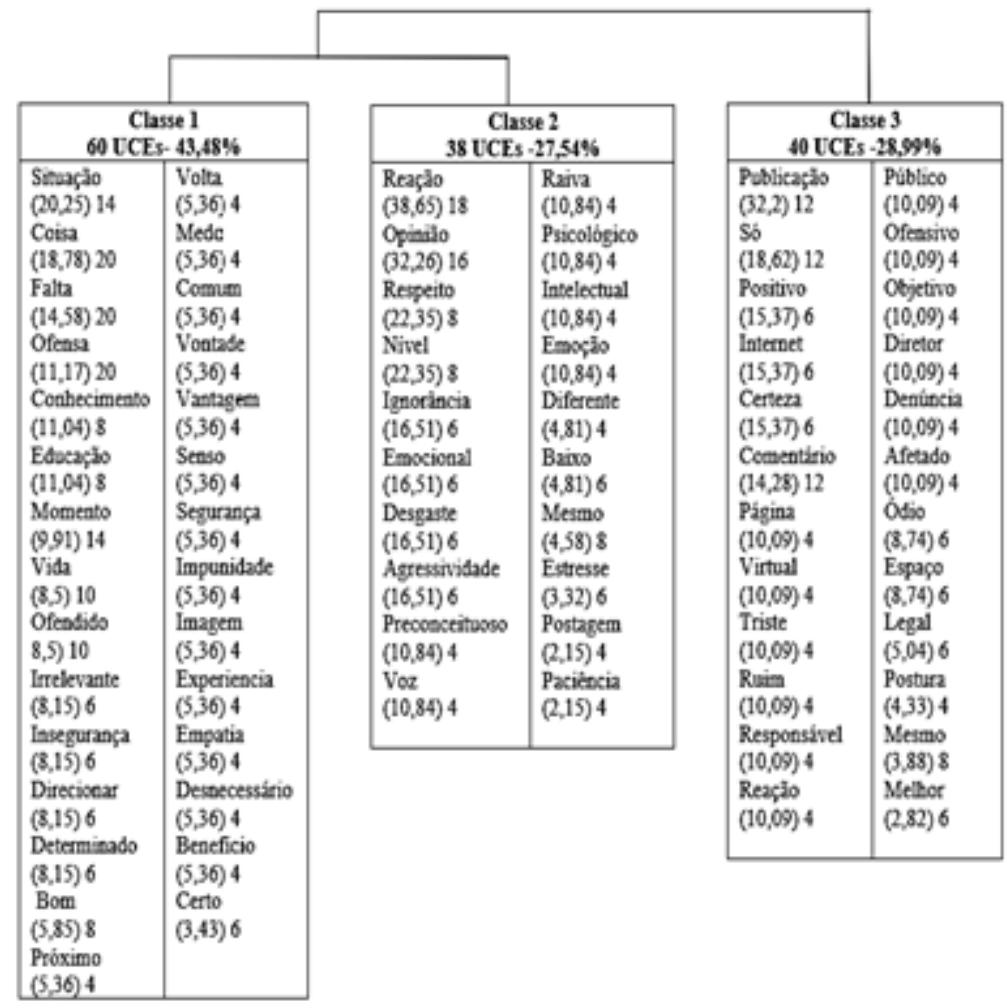

Fig. 1. Dendograma das formas de resposta ao cyber-hate no Facebook

A classe 1, nomeada de "Crenças Comportamentais", obteve o maior aproveitamento (43,5\% das UCE's) e indicou como as pessoas percebem vantagens e desvantagens em reagir ao cyber-hate. Reagir aos insultos foi apreendido como positivo no sentido em que possibilita uma reflexão sobre a interação virtual mediada pelo apoio em rede e a conscientização do papel danoso desse tipo de conduta: "(...) ajuda na saúde mental das outras pessoas, impedindo que elas ofendam de volta ou ofendam outras pessoas que não têm nada a ver com o assunto." (R. 14); “(...) a pessoa está desabafando o que está sentindo e/ou tentando mostrar para a pessoa que ofendeu que aquela atitude não é legal e pode prejudicar muito a vida/psicológico das pessoas." (R. 21). Por outro lado, foi indicado como negativo reagir às ofensas, na medida em que a oposição ao conteúdo odioso possibilita a reprodução do conflito e sua difusão, o que em nada auxilia na promoção de relações virtuais saudáveis: "Não há vantagens, pois, você já passou pela ofensa" (R. 7). "(...) isso pode acabar sendo levado pra outros espaços da vida. 
Gerando uma frieza desnecessária em muitas situações, assim como também ignorando coisas que podem ser necessárias ouvir"; "Reagir a ofensas faz com que os níveis de ansiedade e estresse aumentem, e no final mesmo você dando voz a sua opinião e influenciando a outros, a pessoa central continuará a repetir o mesmo comportamento." (R. 4)

A classe 2, nomeada de "Crenças de Controle" (27,5\% das UCE's), evidenciou aspectos que podem facilitar ou dificultar a reação das vítimas ao cyber-hate. O papel da denúncia, o conhecimento sobre os direitos e até a importância de saber se portar de forma adequada frente às ofensas foram citados como referência ao que predispõe a expressão de reações: "As possibilidades de denúncia facilitam tais reações" (R. 14); "Não ter argumentos sólidos, não ter paciência para as repetições da ignorância cotidiana" (R. 4); "Dificulta em criar uma consciência crítica quando se trata dos comentários e postagens nas redes, impossibilitando o respeito e a diversidade de opiniões" (R. 15).

Por fim, a classe 3 (28,99\% das UCE's), expressa as reflexões dos usuários sobre o julgamento que recebem de terceiros ao responderem ao cyber-hate. Essa classe foi nomeada de "Crenças Normativas" e reporta uma perspectiva mista que gira em torno dos sentimentos de aprovação por familiares e amigos ou da desaprovação da manifestação de reação aos discursos de ódio. Foi indicado também por alguns respondentes um sentimento de indiferença ou de autonomia frente à opinião alheia acerca de suas condutas nas redes sociais: "No geral, acredito que a minha reação seja julgada como positiva, já que o meu objetivo é legal e está dentro das diretrizes da rede social" (R. 10); "Acham que eu deveria retribuir de forma agressiva." (R. 23); "Depende da minha reação. Se eu responder de forma vulgar, é algo imoral." (R. 30); "Não sei e na Realidade não me importa!" (R. 25).

\section{Discussão}

O presente estudo objetivou investigar as formas de reação das vítimas de cyber-hate no Facebook frente à intolerância digital. Os resultados das análises textuais possibilitaram identificar três classes temáticas que podem ser entendidas como eixos organizadores das formas de reação ao cyber-hate, fundamentados, por sua vez, a partir da Teoria da Ação Planejada (TAP): (1) crenças comportamentais; (2) crenças de controle e (3) crenças normativas. As crenças comportamentais se configuram como o componente avaliativo das atitudes e das consequências da realização do comportamento alvo. As crenças de controle descrevem a percepção de gerenciamento que as pessoas possuem para controlar a emissão do comportamento alvo. As crenças normativas se referem às normas subjetivas derivadas da influência social para execução do comportamento alvo (Fernandes et al., 2019). A partir dos resultados apresentados, pode-se afirmar que o objetivo do presente estudo tenha sido alcançado, indicando, em geral, que as crenças exercem um papel central nas formas de resposta de vítimas ao cyber-hate e que o amparo teórico e conceitual da TAP se constituiu como um pertinente dispositivo para a investigação deste fenômeno corroborando com estudos recentes no contexto brasileiro (Chaia \& Brugnago, 2014; Marques-Pinto, Alvarez e Pereira, 2018; Fernandes, Nascimento, Pereira, Melo \& Carlos, 2020).

Em linhas gerais, foi possível identificar que as pessoas tendem a revelar mais desvantagens ao refletir sobre as formas de reação às ofensas nesta rede social, como por exemplo, o receio de que a postura nas redes extrapole o ambiente virtual e tome proporções danosas na vida real, resultando em algum tipo de prejuízo ou até implicações sociais e judiciais. No entanto, as evidências demonstraram também que os respondentes percebem a reação das vítimas como propulsora para a aceitação e respeito à diversidade, sendo fundamental para assegurar e legitimar os direitos das minorias. Nessa lógica, a utilização de premissas sólidas para responder ao cyber-hate foi apontada como necessária para a promoção de relações virtuais adequadas. 
Esse dado denota que o uso do Facebook, principalmente, no Brasil, acompanha problemáticas dignas de análise e relevância científica, uma vez que permite esclarecer elementos importantes da dinâmica de utilização dessa rede social bem como, as formas através das quais as pessoas se expressam neste ambiente e seus impactos na vida real.

No contexto das vítimas de cyber-hate, de acordo com Marques-Pinto e Alvarez (2016), as redes sociais online se tornaram mais propensas à propagação de grupos que assumem discursos de ódio, através da defesa de ideais de cunho xenófobo, racista e homofóbico, que procuram recrutar adeptos, caracterizando um contexto aversivo aos grupos minoritários. Portanto, trata-se de um espaço não vantajoso para livre expressão que, frequentemente, viabilizada pela falta de empatia e pela banalização de qualquer tipo de intolerância, seja nos contextos off-line ou online (Botti, 2019), permite que esse espaço seja palco da disseminação do ódio.

Apesar da Constituição Federal do Brasil de 1988, limitar a liberdade de expressão sobre discursos de ódio e vedar a manifestação de forma anônima (Brasil, 1988), o direito como regulador das relações humanas, encontra dificuldades em acompanhar as novidades advindas das tecnologias, e com isso não consegue efetivamente punir os crimes cometidos nesse meio, o que acaba sendo um facilitador para esse tipo de crime (Freitas \& Santos, 2019).

A propagação do ódio, segundo Martins (2019), é composta por duas facetas: o insulto e a instigação. Na primeira há a propagação do insulto propriamente dito, e na segunda há a instigação para o compartilhamento e 0 apoio de curtidas de outras pessoas compactuantes com a ideia. No Brasil, regidos por uma falsa pacificidade e cordialidade, observa-se que a negação, a negligência e a indignação seletiva são as principais estratégias de enfrentamento ao discurso de ódio (Hoepfner, 2014).

Diante do exposto, reitera-se que a situação das vítimas de cyber-hate ainda é crítica no Brasil, principalmente no ambiente do Facebook, por se tratar da rede social mais utilizada do mundo. Tanto é, que o próprio Facebook instituiu normas mais rigorosas em relação a perfís proliferadores de discursos de ódio e discriminação na sua comunidade virtual (Facebook, 2020). Não obstante, apesar desta postura por parte da empresa Facebook, o que pode ser considerado um avanço em termos de políticas de combate e controle aos conteúdos dessa natureza, essa rede social online, ainda se configura como um espaço virtual de expressão de ódio e intolerância, a exemplo da intolerância racial frequentemente expressa no Facebook, como apontou Fernandes et al. (2020).

Acrescenta-se ainda a necessidade de atentar aos efeitos dos hábitos de uso do Facebook no cotidiano de seus usuários. Marques-Pinto et al. (2018), considerando amostras do Brasil e Portugal, demostraram que em geral, jovens menos ajustados psicossocialmente são mais propensos a correr riscos e a aproveitar oportunidades. Tendo sido encontradas evidências de que o tempo de uso do Facebook está relacionado ao maior nível de exposição ao risco na amostra de lusitanos, contudo, os autores reforçam a necessidade de se considerar fatores psicológicos, socioculturais e de desenvolvimento envolvidos neste contexto, tal moderação não foi identificada na amostra de brasileiros.

\section{Conclusões}

A análise das crenças que subjazem o comportamento de reagir aos insultos e os resultados dela provenientes auxiliam na compreensão da configuração das formas de reação ao cyber-hate na rede social analisada. Nesse sentido, a ocupação do cyberespaço por grupos minoritários amplia as possibilidades de transformação social, visto que, anteriormente à época das RSO, a propagação da informação era de monopólio de poucos, o que dificultou historicamente as minorias sociais de defenderem suas agendas de reivindicações (Mittmann, 2011). 
Nesta direção, o presente estudo se reveste de relevância científica e social, uma vez que a metodologia selecionada para esta investigação traz à tona a importância de dar voz às vítimas de cyber-hate, e aponta para necessidade de que em uma via de mão dupla, ou seja, tanto no ambiente virtual quanto real, o reconhecimento do usuário enquanto vítima do discurso de ódio, auxilie no desenvolvimento de estratégias para combatê-lo. Por fim, conclui-se que identificar essa prática como uma tipologia de violência que se utiliza da exposição das fraquezas e dos estigmas sociais da vítima favorece a legitimação do cyberhate e da sua popularidade negativa (Amaral \& Coimbra, 2015).

Finalmente, apesar deste estudo responder aos objetivos satisfatoriamente, há algumas limitações que devem ser mencionadas. Ressalta-se, primeiramente, o número restrito de participantes do presente estudo, fato que inviabiliza toda e qualquer generalização para o ambiente virtual brasileiro, e nem mesmo para a comunidade do Facebook na sua totalidade, tendo em vista a diversidade desta rede social. Logo, espera-se que estudos futuros realizem inquéritos mais amplos e com participantes diversificados, uma vez que, estudos dessa natureza são essenciais para preencher lacunas existentes na literatura da área, principalmente no contexto brasileiro.

Seria importante também considerar outros ambientes virtuais (e.g. Instagram, Twitter, TikTok, etc) para uma análise mais abrangente deste fenômeno que têm estado expressivamente presente no cotidiano brasileiro, principalmente, no contexto da pandemia da covid-19 que, em função das medidas de restrições sociais recomendadas pela autoridades sanitárias e de saúde, têm colocado centralidade da virtualidade em diversas esferas das nossas vidas.

\section{Referências}

Ajzen, I. (1991). The theory of planned behavior. Organizational behavior and human decision processes, 50(2), 179-211.

Ajzen, I., \& Driver, B. L. (1991). Prediction of leisure participation from behavioral, normative, and control beliefs: An application of the theory of planned behavior. Leisure sciences, 13(3), 185204.

Ajzen, I., \& Fishbein, M. (2005). The Influence of Attitudes on Behavior. In D. Albarracín, B. T. Johnson, \& M. P. Zanna (Eds.), The handbook of attitudes (p. 173-221). Lawrence Erlbaum Associates Publishers.

Amaral, A., \& Coimbra, M. (2015). Expressões de Ódio nos Sites de Redes Sociai: O Universo dos Haters no caso \#eunãomereçoserestuprada. Contemporanea, 13(1), 294-310.

Botti, N. C. L. (n.d.). Desafios Éticos e Suicídio na Pós-Modernidade. Cadernos Zygmunt Bauman, 9, 2-20.

Brasil. (1988). Constituição da república federativa do Brasil. Senado Federal, Centro Gráfico.

Camargo, B. V.; Justo, A. M. (2016) Tutorial para uso do software IRAMUTEQ. Laboratório de Psicologia Social da Comunicação e Cognição: Universidade Federal de Santa Catarina.

Chaia, V., \& Brugnago, F. (2014). A nova polarização política nas eleições de 2014: Radicalização ideológica da direita no mundo contemporâneo do Facebook. Aurora. Revista de Arte, Mídia e Política, 7, 99-129. Disponível em https://revistas.pucsp.br/index.php/aurora/article/view/22032

Facebook. (2021). Facebook newsroom: company info. Disponível em: http://newsroom.fb.com/company-info/. Acessado em 17 de março de 2021.

Fernandes, Sheyla, Nascimento, Marcikele, Pereira, Alanda, Melo, Erika, \& Carlos, Kathleen. (2020). Relações raciais digitais: um estudo sobre as formas de expressão da intolerância racial no Facebook. RISTI - Revista lbérica de Sistemas e Tecnologias de Informação, 38, 79-91. https://dx.doi.org/10.17013/risti.38.79-91

Fishbein, M., \& Ajzen, I. (2011). Predicting and changing behavior: The reasoned action approach. Taylor \& Francis.

Francis, J., Eccles, M. P., Johnston, M., Walker, A. E., Grimshaw, J. M., Foy, R., ... \& Bonetti, D. (2004). Constructing questionnaires based on the theory of planned behaviour: A manual for health services researchers. 
Freitas, L. C., \& Santos, J. J. (2019). Dos Crimes Virtuais Cometidos Se Utilizando do Anonimato da Deep Web (pp. 1-19). ETIC 2019.

Hoepfner, S. (2014). Apontamentos sobre a questão ético-midiática do discurso de ódio na rede social. Esferas, 5(4). https://doi.org/10.19174/esf.v0i4.5264

Junior, J. A. S. (2018). Análise Dos Preditores Da Intenção De Utilizar a Rede Social Facebook: Uma Aplicação Da Teoria Da Ação Planejada. In UFAL - Universidade Federal De Alagoas.

Marques, T. P., Marques-Pinto, A., \& Alvarez, M.-J. (2016). Estudo psicométrico da Escala de Avaliação dos Riscos e Oportunidades dos jovens utilizadores do Facebook. Revista Iberoamericana de Diagnóstico e Avaliação Psicológica, 41, 145-158.

Marques, T., Marques-Pinto, A., Alvarez, M., \& Pereira, C. (2018). Facebook: Risks and Opportunities in Brazilian and Portuguese Youths with Different Levels of Psychosocial Adjustment. The Spanish Journal of Psychology, 21, E31. doi:10.1017/sjp.2018.34

Martins, A. C. L. (2019). Hate speech in social networks and recognition of the other: The M. case. Revista Direito GV, 15(1), 1-30. https://doi.org/10.1590/2317-6172201905

Mello e Marques, N. E., \& Nobre, T. L. (2021). Uma reflexão sobre o discurso de ódio nas redes sociais brasileiras. IROCAMM, 1(4), 73-88.

Mittmann, S. (2011). Alguns apontamentos sobre militância digital. Ed. Universitária, 119-139.

Pelbart, P. (2019). O mimimi como categoria biopolítica. Cadernos de Subjetividade, 13(20), 99-104.

Quandt, T., \& Festl, R. (2017). Cyberhate. The international encyclopedia of media effects, 1-8.

Ramos, M. G., Lima, V. M. R., \& Amaral-Rosa, M. P. (2018). Contribuições do software IRAMUTEQ para a Análise Textual Discursiva. Congresso Ibero-Americano Em Investigação Qualitativa, 1, 505-514.

Roazzi, A., Almeida, N. D., Souza, B. C., \& Roazzi, M. (2014). Da Teoria da Ação Racional à Teoria da Ação Planejada: Modelos para explicar e predizer o comportamento. Revista Amazônica, 13(1), 175-208.

Rocha, J. L. A. D., \& Mendes, A. P. T. (2020). Cartilha de orientação para vítimas de discurso de ódio. Fundação Getúlio Vargas.

Rosado, L. A. S., \& Tomé, V. M. N. (2015). As redes sociais na internet e suas apropriações por jovens brasileiros e portugueses em idade escolar. Revista Brasileira de Estudos Pedagógicos, 96(242), 11-25.

Silva, L. R. L., \& Botelho-Francisco, R. E. (2020). Gestão de conteúdo de Ódio no Facebook: um estudo sobre haters, trolls e naysayers. P2P E Inovação, 6, 38-56.

Souza, M. A. R., Wall, M. L., Thuler, A. C. M. C., Lowen, I. M. V., \& Peres, A. M. (2018). O uso do software IRAMUTEQ na análise de dados em pesquisas qualitativas. Revista Da Escola de Enfermagem Da USP, 52, 1-7.

Statista. (2021). Número de usuários ativos mensais de Facebook em todo o mundo a partir do 40 trimestre de 2020 (em milhões). https://www.statista.com/statistics/264810/number-of-monthlyactive-facebook-users-worldwide/

Zenha, L. (n.d.). Redes sociais online: o que são as redes sociais e como se organizam? Caderno de Educação, 49, 19-42. 